\title{
Leishmania tropica infection in wild and domestic canines
}

\author{
G Baneth $^{1 *}$, A Nasereddin $^{2,3}$, Z Abdeen ${ }^{2}, \mathrm{CL}$ Jaffe $^{3}$ \\ From The 1st Conference on Neglected Vectors and Vector-Borne Diseases (EurNegVec): with Management \\ Committee and Working Group Meetings of the COST Action TD1303 \\ Cluj-Napoca, Romania. 8-11 April 2014
}

Leishmania tropica is a causative agent of cutaneous leishmaniasis in the Middle East, North Africa and some parts of southeastern Europe. It has also been described as a cause of human visceral leishmaniasis. Although cutaneous leishmaniasis caused by L. tropica is usually considered an anthroponotic infection transmitted between people directly by phlebotomine sand flies without the involvement of an animal reservoir, in Israel, Jordan and the Palestinian Authority it is a zoonosis with the rock hyrax (Procavia capensis) as a main reservoir host. Golden jackals (Canis aureus) and red foxes (Vulpes vulpes) have also been found to be infected with L. tropica in Israel and are assumed to have a role in spreading the infection to distant locations, but clinical signs of infection in these wild canids have not been detected.

In the domestic dog, L. tropica infection has been reported in only a few cases from Morocco and Iran where infection was mostly described as involving the visceral organs. While some surveys describe the detection of parasite infection from dog organs by culture or PCR without much detail on the manifestations of disease, reports from Morocco described two dogs infected with L. tropica with clinical manifestations similar to those found in canine L. infantum infection including generalized lymphadenomegaly, onychogryphosis, alopecia, keratoconjunctivitis, and also glomerulonephritis in one case. A report from northwestern Iran also described $L$. tropica in a dog with cutaneous and visceral involvement comparable to canine $L$. infantum infection. A young dog from a focus of L. tropica human cutaneous leishmaniasis near Jerusalem, with a large proliferative red mucocutaneous lesion between the upper lip and the nose was recently found to have mucocutaneous leishmaniasis caused by L. tropica.

In conclusion, domestic and wild canine infection with L. tropica may be more prevalent in areas of endemic human $L$. tropica cutaneous leishmaniasis than currently recognized, and canines should be evaluated as possible additional reservoirs for human infection.

\section{Funding}

This study was supported by supported by the U.S. Agency for International.

Development (USAID) Middle East Regional Cooperation Program (MERC) project TA-MOU-08-M27-072 and by the Deutsche Forschungsgemeinschaft (DFG) as part of a German-Israeli-Palestinian cooperative project $\mathrm{SCHO}$ 448/8-1.

\begin{abstract}
Authors' details
${ }^{1}$ School of Veterinary Medicine, Hebrew University, P.O. Box 12, Rehovot 76100, Israel. ${ }^{2} \mathrm{Al}-\mathrm{Quds}$ Nutrition and Health Research Institute, Al-Quds University, Abu Deis, Palestinian Authority. ${ }^{3}$ Department of Microbiology and Molecular Genetics, IMRIC, Hebrew University-Hadassah Medical School, Jerusalem, Israel.
\end{abstract}

Published: 1 April 2014

doi:10.1186/1756-3305-7-S1-O27

Cite this article as: Baneth et al:: Leishmania tropica infection in wild and domestic canines. Parasites \& Vectors 2014 7(Suppl 1):O27.

\footnotetext{
* Correspondence: gad.baneth@mail.huji.ac.il

'School of Veterinary Medicine, Hebrew University, P.O. Box 12, Rehovot

76100, Israel

Full list of author information is available at the end of the article
}

(c) 2014 Baneth et al.; licensee BioMed Central Ltd. This is an Open Access article distributed under the terms of the Creative Commons 